\title{
THE FORMATION OF DISKS BY INELASTIC COLLISIONS OF GRAVITATING PARTICLES
}

\author{
Applications to the Formation of Galaxies \\ ANDRÉ BRAHIC \\ Observatoire de Paris-Meudon, Université de Paris VII, France
}

\begin{abstract}
The numerical study of a gravitating system of colliding particles has many potential applications, for instance the formation of flat galaxies, the formation of the solar system and the evolution of Saturn's rings. Preliminary results are presented for the galactic case. The system tends towards a final equilibrium state and it seems that such a collision mechanism can flatten a protogalaxy.
\end{abstract}

Since the time of Descartes, Laplace and Poincaré, turbulence has played a central role in cosmological speculation and in theories of the formation of galaxies. It appears not unreasonable to suppose that the early Universe was statistically uniform on a macroscopic scale - as suggested by the microwave background - but disordered on the small scale. Therefore we shall assume that a protogalaxy consists of a small number of clouds or turbulence eddies, whose positions and velocities are initially randomly distributed. Each cloud describes an orbit in the galactic field and the system loses energy through inelastic collisions between clouds.

Poincare (1911) showed qualitatively that in such a system the following things happen:

(1) a central condensation will be formed and, in order to conserve angular momentum, the outer part of the system will expand;

(2) the system as a whole will flatten into a plane perpendicular to the initial angular momentum vector;

(3) the orbits of the bodies will become increasingly more circular.

Brosche (1970) made a quantitative and analytical model of this process with some approximations and found that the Hubble sequence could be thought of as an angular momentum sequence at constant mass. He also noted that a detailed $N$ body calculation could be expected to improve this kind of model.

With the help of M. Hénon, I am at present developing a general program for this kind of problem. It is a numerical simulation of a gravitating system of particles with inelastic collisions. Such a program has many potential applications other than the formation of galaxies, for instance the dynamics of Saturn's rings, the formation of the solar system and also the evolution of the nuclei of galaxies. We may note other work in this context: by Spitzer and Saslaw (1966), Spitzer and Stone (1967) and Sanders (1970) who have studied the nuclei of galaxies; McCrea (1960), Alfvén and Arrhenius (1970) and Urey (1972) who have studied the formation of the solar system and planets; and Jeffreys (1947), Bobrov (1970) and Cook et al. (1972) who have studied Saturn's rings.

Calculations of this sort have so far only been carried out by Ulam (1968) and by 

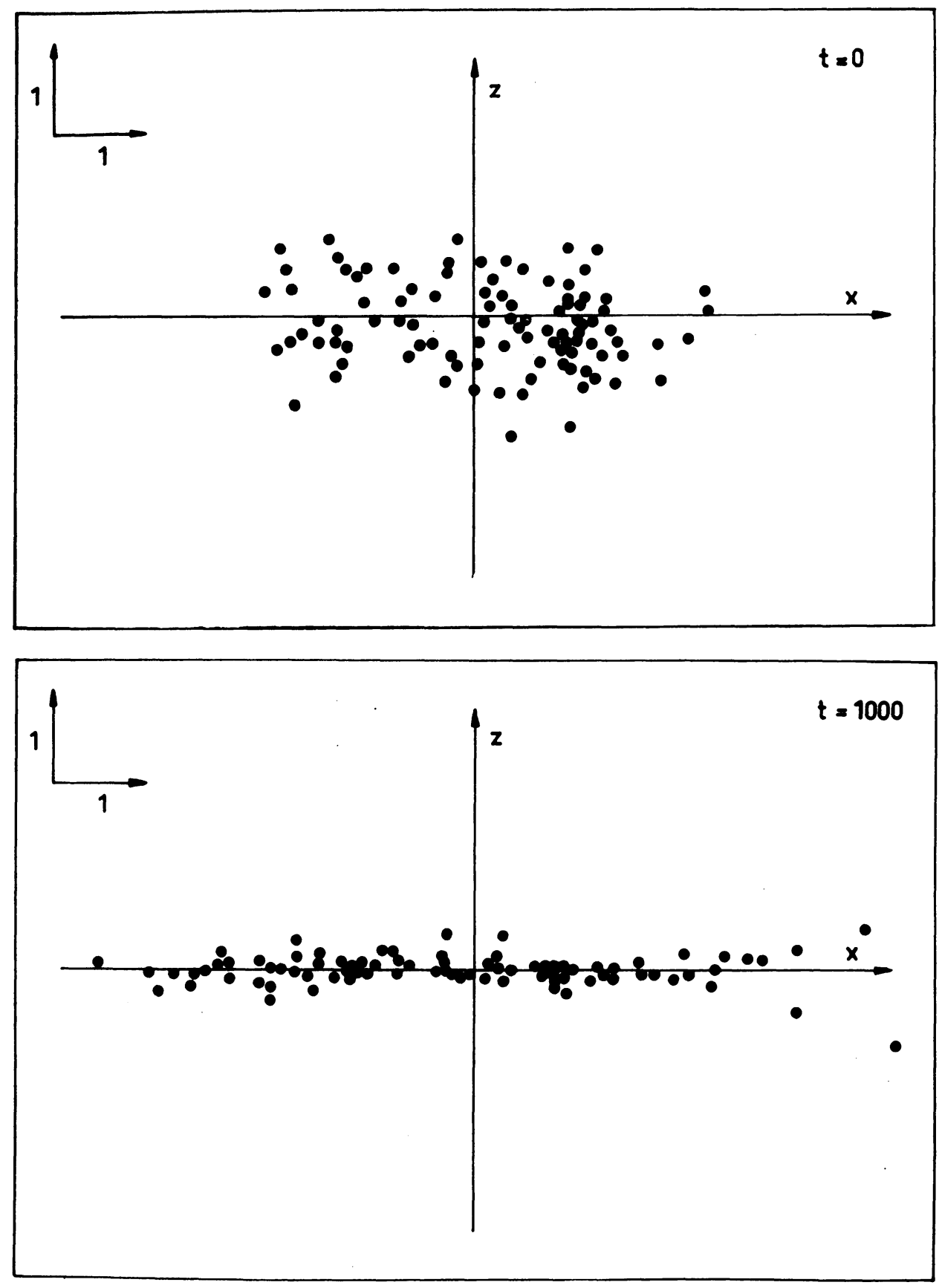

Fig. 1. Projections onto a plane parallel to the initial angular momentum vector at the initial time and at the end of the computation. The number $N$ of clouds is equal to 100 . Each cloud has the same radius $R=0.07$ and the same mass. The coefficient of elasticity $k$ is equal to 0.3 . The maximum value of the initial inclinations of the orbits is equal to 0.5 radian. The unit of time corresponds approximately to the mean time necessary for a cloud to move through one radian on its orbit. After 2500 collisions, one out of 100 clouds has escaped and sixteen out of 100 clouds have fallen on the central body of radius 0.1 . 

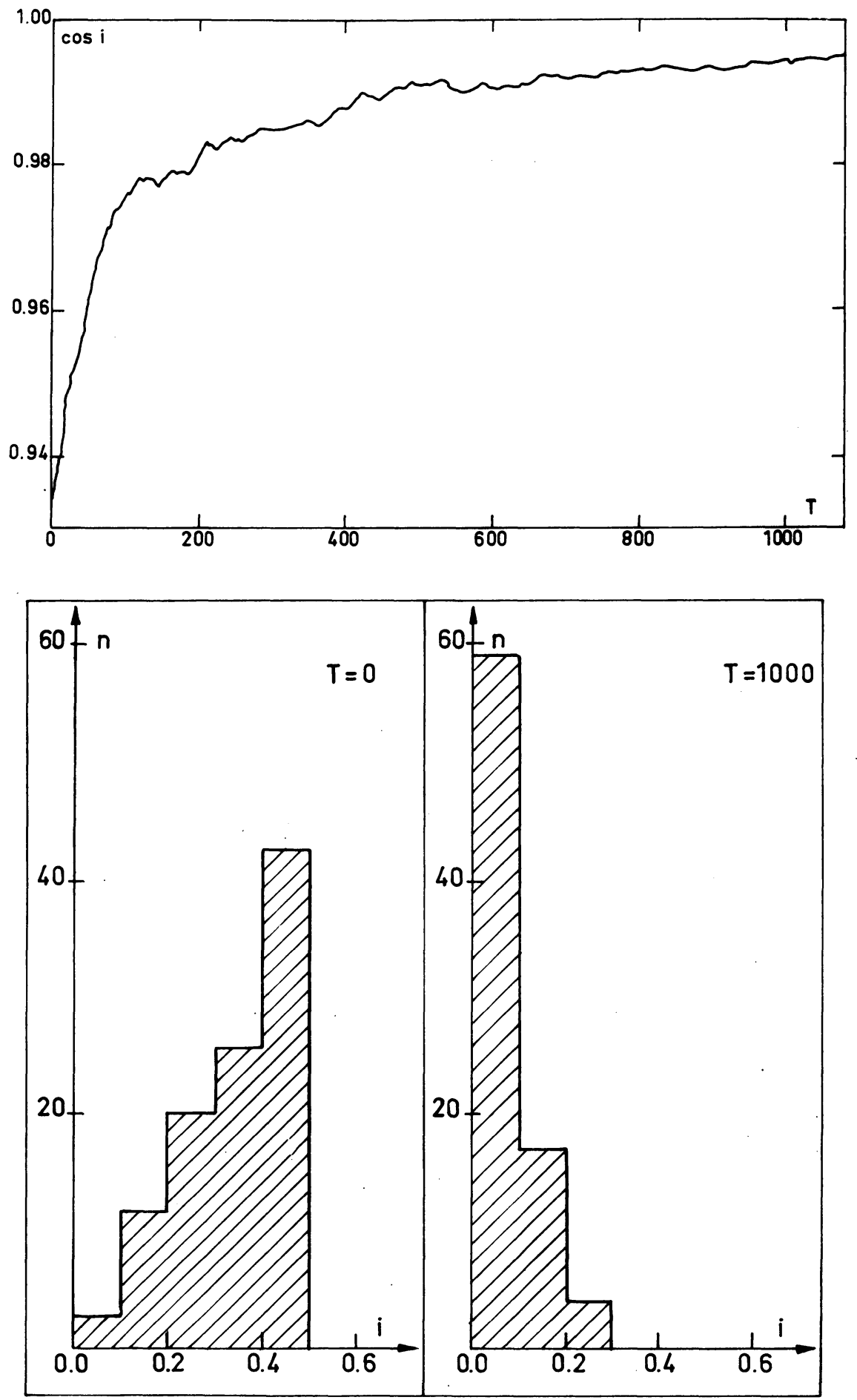

Fig. 2. Top: The variation as a function of time of the mean cosine of the inclination $i$. Bottom: Histograms of $i$ at the initial time and at the end of the computation.

https://doi.org/10.1017/S0074180900024426 Published online by Cambridge University Press 
Trulsen (1972). Ulam was interested in the nuclei of galaxies; he used a small number of bodies and, moreover, assumed that they coalesced into a single body after collision. Trulsen studied the dynamics of jetstreams and the formation of planets. Corresponding numerical experiments have been made in molecular dynamics by Alder and Wainwright (1959, 1960), Rahman (1964) and Verlet $(1967,1968)$ and have stimulated significant progress. A very similar approach has also been applied by us to the case of Saturn's rings (Brahic 1973).

We thus consider a system of clouds moving in a given gravitational field and interacting through inelastic collisions. We neglect the mutual attraction of the clouds and we study numerically the evolution of the system. I shall not here enter into the technical details of the rather intricate calculations, which will be given in a paper in preparation. Let me say only that the principal difficulty is to know whether two clouds will in fact collide. This problem is comparatively simple in molecular dynamics because particles move in straight lines between collisions, but in our case each cloud describes a galactic orbit.

The time scale $t_{\mathrm{c}}$ of evolution of the system is of the order of the mean time between collisions for one cloud. Therefore it is inversely proportional to the number $N$ of clouds and to their geometrical cross-section which is itself proportional to the square of the mean radius $R$ of a cloud. In fact, if we change either $N$ or $R$, we simply make the evolution go slower or faster. Consequently, a proper choice for $N$ and $R$ permits us to follow realistically the evolution of our system using a minimum of computer time, albeit using a reduced number of larger bodies. However, the procedure becomes unsatisfactory is $R$ is too large, since the gravitational force would then not be the same for two colliding particles, thereby introducing distortion which would increase towards the centre of the system. Too small a number of clouds would also be unsatisfactory because statistical fluctuations would be too large.

I am currently studying a sequence of numerical models and investigating their astrophysical implications. Results have been obtained for the simplest case in which cloud orbits are keplerian around a central mass point. Positions and velocities at any given time are obtained from Kepler's equation. In a collision the grazing component of velocity is conserved and the perpendicular component is multiplied by a coefficient $k$ which lies between 0 corresponding to the completely inelastic case and 1 corresponding to the elastic case. The initial conditions were set up by selecting at random the six elements of the keplerian orbit in such a way that trajectories were all ellipses lying between two spheres centred on the central mass point and with inclinations lying between 0 and some maximal value. We have assumed that clouds on hyperbolic trajectories escape at once and, for technical reasons, that clouds near to the centre are captured by the centre of mass.

The four figures show an example of the evolution of the system. We can see in Figure 2 that the system has flattened considerably after 2500 collisions, but it is not yet completely flat. Figure 2 shows the change of inclination with time. Figure 3 shows a spread of radius: the system extends. We can see in Figure 4 that, at first, the eccentricities increase because a thermal equilibrium is established between radial and 

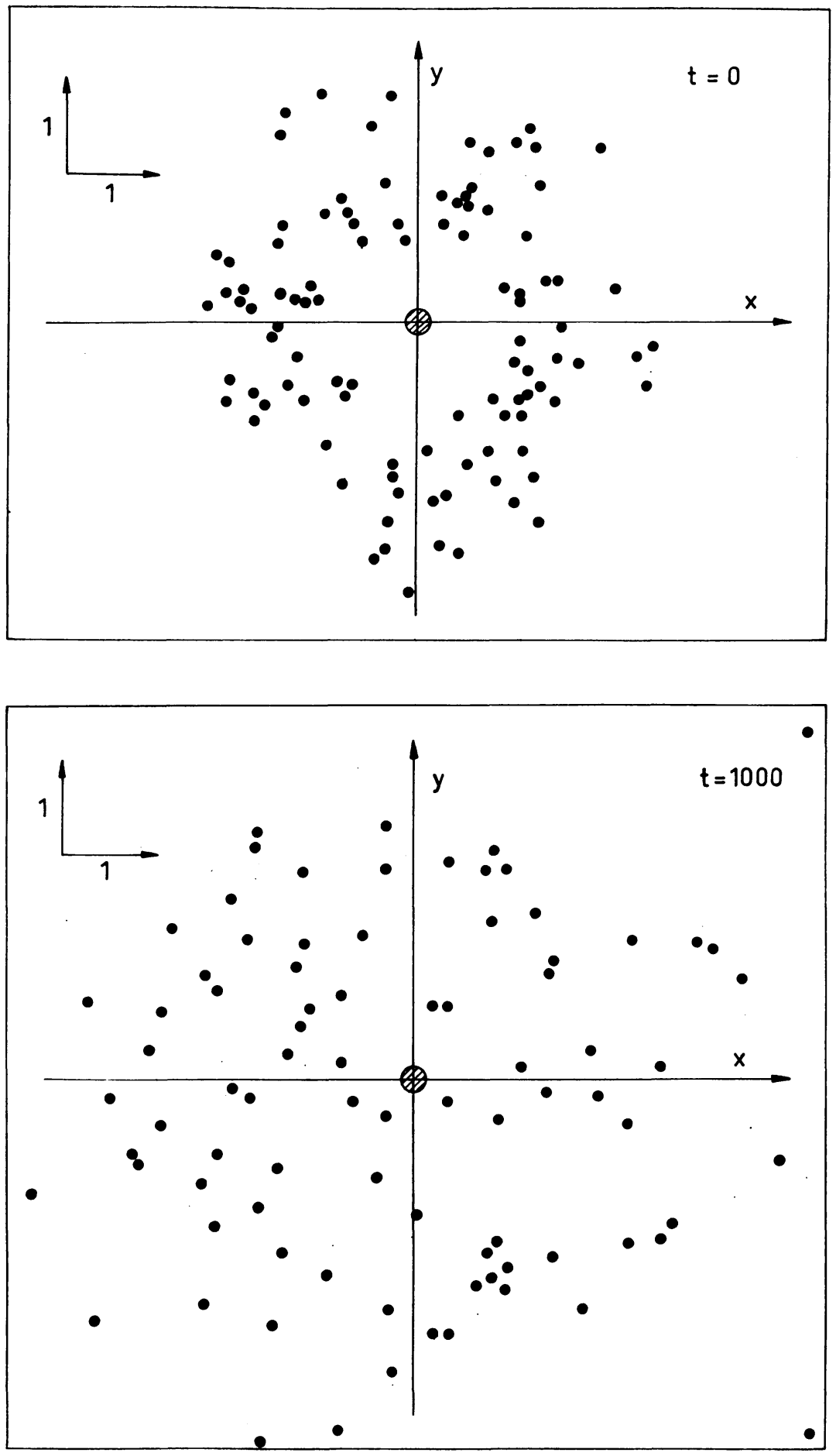

Fig. 3. Projections onto a plane perpendicular to the initial angular momentum vector. Initial trajectories are all ellipses lying between two spheres of radius 1 and 3 respectively, centred on the central mass point. 

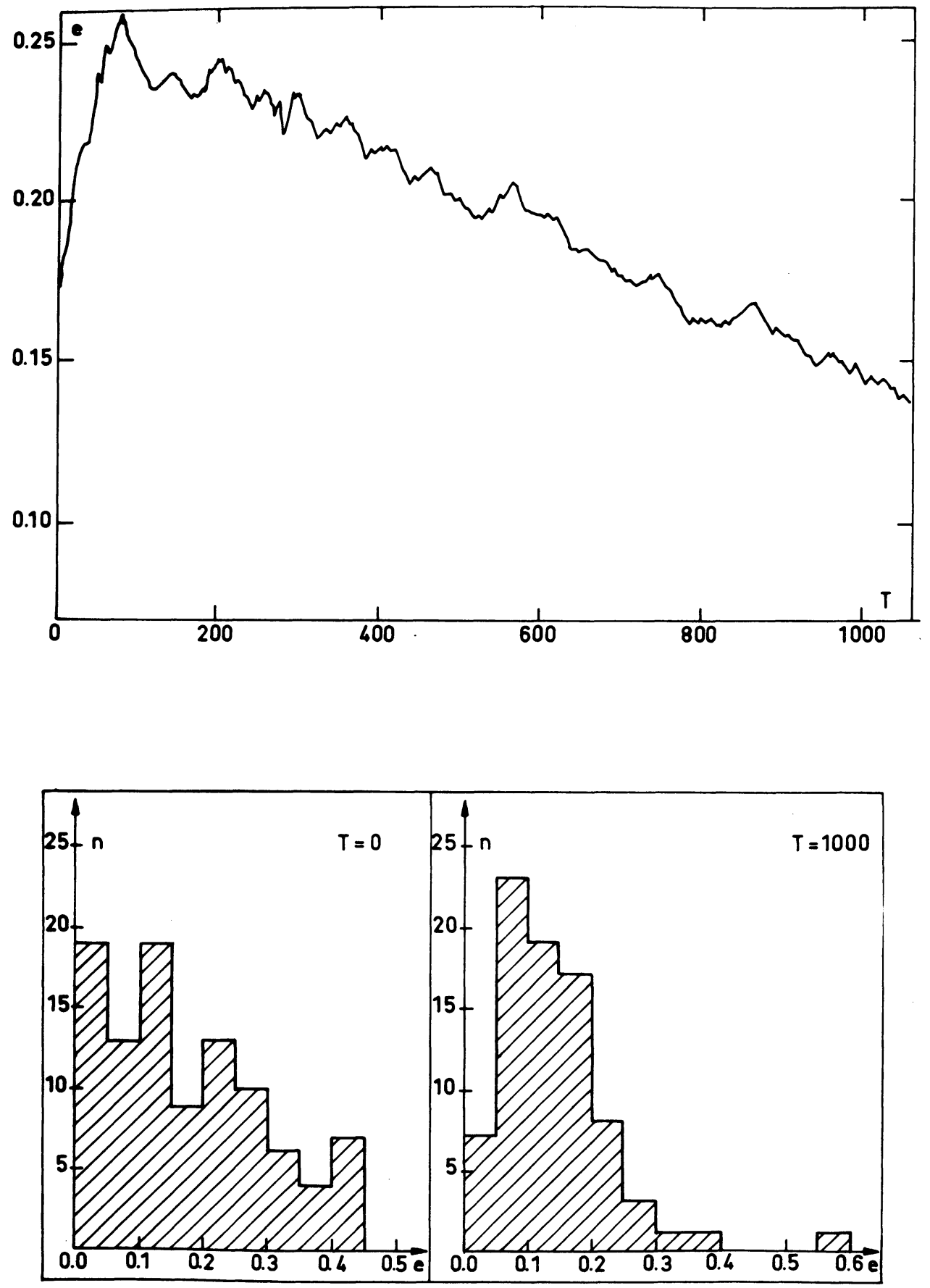

Fig. 4. The variation as a function of time of the mean eccentricity of the orbits and the corresponding histograms. 
vertical velocities. After that the orbits tend to become more and more circular.

Of course, this first model is probably too crude for a realistic description of a galaxy. But the results already suggest that:

(1) the collision rate decreases with time and the system tends towards a final equilibrium state;

(2) such a mechanism can flatten a protogalaxy. The flattening is already important when each cloud has suffered about ten collisions.

Subsequent models will include fragmentation of clouds during each collision, and also the use of a more realistic field.

\section{References}

Alder, B. J. and Wainwright, T. E.: 1959, J. Chem. Phys. 31, 459.

Alder, B. J. and Wainwright, T. E.: 1960, J. Chem. Phys. 33, 1439.

Alfvén, H. and Arrhenius, G.: 1970, Astrophys. Space Sci. 8, 338; 9, 3.

Bobrov, M. S.: 1970, in Surfaces and Interiors of Planets and Satellites, Academic Press, London and New York, p. 377.

Brahic, A.: 1974, in Y. Kozai (ed.), 'The Stability of the Solar System and of Small Stellar Systems', IAU Symp. 62, 83.

Brosche, P.: 1970, Astron. Astrophys. 6, 240.

Cook, A. F., Franklin, F. A., and Palluconi, F. D.: 1973, Icarus 18, 317.

Jeffreys, H.: 1947, Monthly Notices Roy. Astron. Soc. 107, 260.

McCrea, W. H.: 1960, Proc. Roy. Soc. London A256, 245.

Poincaré, H.: 1911, Leçons sur les hypothèses cosmoniques, Hermann, Paris, Chapter 5, p. 86

Rahman, A.: 1964, Phys. Rev. 136A, 405.

Sanders, R. H.: 1970, Astrophys. J. 159, 1115.

Spitzer, L. and Saslaw, W. C.: 1966, Astrophys. J. 143, 400.

Spitzer, L. and Stone, M. E.: 1967, Astrophys. J. 147, 519.

Trulsen, J.: 1972, Astrophys. Space Sci. 17, 241; 17, 330; 18, 3.

Ulam, S. M.: 1968, Bull. Astron. 3, 265.

Urey, H. C.: 1972, Astrophys. Space Sci. 16, 311.

Verlet, L.: 1967, Phys. Rev. 159, 98.

Verlet, L.: 1968, Phys. Rev. 165, 201.

\section{DISCUSSION}

Abell: Can you scale your calculations to an actual galaxy? That is, with conditions you might expect for a protogalaxy, how long would those ten or so collisions (per condensation) take to flatten a system of galactic mass and dimensions?

Brahic: The only thing which can be said is that the flattening is important when every cloud has experienced about ten collisions. The mean number of collisions experienced by one cloud depends on the initial number and dimension of the clouds. It seems reasonable to assume that in a realistic case each cloud will experience one to four collisions per revolution. In that case, the flattening will occur in about $10^{9} \mathrm{yr}$.

Savedoff: How does the flattening time depend upon the parameter $k$ of your model?

Brahic: For $k$ below 0.4 or 0.5 one gets the same effect, but for $k$ near 0.7 there is no flattening. 